\title{
O Programa Bolsa Família Estimula a Permanência dos Trabalhadores Brasileiros de Baixa Renda no Mercado Informal?
}

\author{
Augusto Mussi Alvim \\ Pontifícia Universidade Católica do Rio Grande do Sul (PUCRS) - Porto Alegre - \\ RS - Brasil \\ ORCID: https://orcid.org/0000-0003-0130-6880 \\ Carlos David Godward \\ Pontifícia Universidade Católica do Rio Grande do Sul (PUCRS) - Porto Alegre - \\ RS - Brasil \\ ORCID: https://orcid.org/0000-0002-8287-3985
}

\begin{abstract}
Resumo
Os Programas de Transferência de Renda Condicionada (PTRCs) mostraram-se eficientes na redução da pobreza e da desigualdade, na melhoria dos índices de escolaridade e de saúde e na inclusão social de uma parcela significativa na sociedade. Todavia, um dos aspectos ainda pouco estudados a respeito dos PTRCs é o potencial destes programas de estimular a permanência dos trabalhadores brasileiros de baixa renda no mercado informal, com o propósito de se manterem "invisíveis" aos gestores do programa e, assim, receberem os benefícios. Para analisar este comportamento no mercado laboral em seis capitais brasileiras foi utilizado um VEC (Vetor de Correção de Erros). Os resultados do estudo mostram que existe uma relação positiva e causal entre o número de beneficiários do bolsa família e o número de pessoas no mercado laboral. Embora estas variáveis tenham uma relação causal positiva e persistente no tempo, os efeitos do Programa Bolsa Família sobre o mercado informal se mostraram pequenos no período analisado.
\end{abstract}

Palavras-chave: Bolsa Família. Programas de Transferência de Renda Condicionada. Mercado de trabalho informal.

\section{Does the Family Grant Program Stimulate the Permanence of Low-Income Brazilian} Workers in the Informal Market?

Abstract

The Conditional Cash Transfer Programs (CCTPs) have been shown to be effective in reducing poverty and inequality, improving education and health, and social inclusion of a significant portion of society. However, one of the aspects still unwell studied about the CCTPs is the potential of these programs to stimulate the permanence of low-income Brazilian workers in the informal market, with the purpose of remaining "invisible" to the program supervisors and thus receiving benefits. To analyze this behavior in the labor market in six Brazilian state's capitals, an Error Correction Vector (VEC) was used. The study 
results show that there is a positive and causal relationship between the number of family allowance beneficiaries and the number of people in the labor market. Although these variables have a positive and persistent causal relationship over time, the effects of the Family Grant Program on the informal labor market were small in the analyzed period.

Keywords: Family Grant Program. The Conditional Cash Transfer Programs. Informal labor market.

\section{¿Estimula el Programa de Subvenciones Familiares la Permanencia de los Trabajadores Brasileños de Bajos Ingresos en el Mercado Informal?}

\section{Resumen}

Se ha demostrado que los Programas de Transferencia Condicional de Efectivo (PTCEs) son efectivos para reducir la pobreza y la desigualdad, mejorar la educación y la salud, y la inclusión social de una porción significativa de la sociedad. Sin embargo, uno de los aspectos aún poco estudiados sobre los PTCEs es el potencial de estos programas para estimular la permanencia de los trabajadores brasileños de bajos ingresos en el mercado informal, con el propósito de permanecer "invisibles" para los supervisores del programa y así recibir beneficios. Para analizar este comportamiento en el mercado laboral en seis capitales del estado brasileño, se utilizó un Vector de corrección de errores (VEC). Los resultados del estudio muestran que existe una relación positiva y causal entre el número de beneficiarios de subsidios familiares y el número de personas en el mercado laboral. Aunque estas variables tienen una relación causal positiva y persistente a lo largo del tiempo, los efectos del Programa de Subsidios Familiares en el mercado laboral informal fueron pequeños en el período analizado.

Palabras clave: Programa de Subvenciones Familiares. Programas de Transferencia Condicional de Efectivo. Mercado laboral informal.

\section{Introdução}

No final dos anos 1990, na América Latina, o Brasil e o México foram os primeiros que inovaram ao introduzir os Programas de Transferência de Renda Condicionada (PTRC) como uma forma de se contrapor ao aumento de desigualdade de renda nestes países. Entre os principais objetivos destes programas de transferência tem-se a proposição de ações conjuntas com o fim de interromper a transmissão intergeracional da pobreza, condicionando os pagamentos ao cumprimento das corresponsabilidades destinadas a desenvolver 0 capital humano nas crianças (Ferro, Kassouf e Levison, 2010; Sugiyama, 2011).

Além da transferência de renda, os PTRCs incluem o atendimento escolar regular, exames de saúde para crianças, mulheres grávidas e mães lactantes, registros completos de vacinação e participação em sessões de treinamento com foco em nutrição e saúde. Os benefícios são pagos em dinheiro, ao invés da entrega de cestas de alimentos, reconhecendo que as famílias estão em melhor posição do que a administração pública, na decisão de como alocar os recursos disponíveis eficientemente. Como forma de ter um uso mais eficaz dos recursos, as transferências são atribuídas às mães, partindo do pressuposto de que as mulheres fazem uma melhor gestão das despesas de bens e serviços ao benefício das crianças, como alimentação, escolaridade e saúde (Ferro, Kassouf e Levison, 2010; Stampini e Tornarolli, 2012). 
Uma série de estudos mostram o sucesso do Programa Bolsa Família (PBF) em promover investimento em capital humano e reduzir o nível de pobreza. Por exemplo, Chein, Andrade e Ribas (2007) apontam melhoras nutricionais das crianças, enquanto Cardoso e Souza (2004) e Pedrozo (2007) mostram que o PBF foi responsável por elevar a frequência escolar das crianças beneficiadas em três pontos percentuais. Ao mesmo tempo, vários estudos têm documentado como os padrões de vida dos beneficiários do PBF evoluíram ao longo do tempo. Brauw et al (2012) fornece evidências da causalidade entre essas mudanças e a participação no PBF em crianças, mulheres e famílias. O programa é muito bem-sucedido ao reduzir a desigualdade de renda e relativamente bem-sucedido em relação a seus objetivos de combater a pobreza no país (Soares, Ribas e Osório, 2010).

Porém, também, há que se levar em conta outros potenciais efeitos do programa sobre o bem-estar social. Uma das críticas mais frequentes na literatura a respeito do PBF é o chamado "efeito-preguiça". A hipótese é que a transferência criaria um desincentivo ao trabalho, principalmente naquelas famílias para as quais só existe o benefício básico e, portanto, não há condicionalidade a ser cumprida. Foguel e Barros (2010), Teixeira (2011), Chein, Andrade e Ribas (2007), Tavares (2008) e Cardoso e Souza (2004) encontraram efeitos demasiadamente pequenos do Programa Bolsa Família sobre a oferta de trabalho de mulheres e homens adultos, para serem considerados relevantes. Há um efeito um pouco maior apenas para mães, que podem chegar a trabalhar quase duas horas a menos, semanalmente, por receberem uma Bolsa Família.

A motivação deste estudo reside no fato dos rendimentos provenientes do trabalho por conta própria, ou mesmo do emprego sem carteira assinada, sejam menos visíveis do que aquele proveniente do emprego com carteira assinada. Nesse caso é possível que o PBF afete mais a escolha sobre a ocupação em que o indivíduo estaria interessado em trabalhar do que a escolha entre trabalhar ou não trabalhar. Com base nisto, o objetivo deste artigo é analisar o efeito do PBF sobre a permanência dos trabalhadores brasileiros de baixa renda no mercado informal, com o propósito de se manterem "invisíveis" aos gestores do programa e, assim, receberem os benefícios.

Neste contexto, a contribuição deste artigo em relação aos demais trabalhos está em analisar a relação entre o PBF, número de trabalhadores no mercado informal e outras variáveis econômicas a partir de séries de tempo. Com um Vetor de Correção de Erros (VEC) é analisada as relações de curto e longo prazo entre as variáveis permitindo avaliar os efeitos e interrelações ao longo do tempo.

Além desta introdução, na segunda seção é estruturada uma revisão sistemática de literatura sobre os programas de transferência de renda condicionado e o Programa Bolsa Família. Na seção seguinte é descrita a metodologia com detalhes dos modelos, testes econométricos e fonte de dados. Ao final do estudo são apresentados os principais resultados e conclusões do trabalho.

\section{Os Programas de Transferência de Renda Condicionado e o Bolsa Família}

Os Programas de Transferência de Renda Condicionada (PTRCs) subsidiam diretamente famílias pobres, transferindo-lhes mensalmente uma pequena soma de dinheiro com a contrapartida de que as famílias cumpram algumas metas de saúde 
e educação, como levar os filhos ao posto de saúde e enviá-los à escola. O programa mexicano apresentou resultados tão satisfatórios que logo muitos quiseram imitálo. Segundo Saavedra (2016) na América Latina e Caribe existem 26 programas transferência condicionada de renda em operação.

Além de aumentar o número de países com PTRCs, também aumentou o número de famílias contempladas e o volume de recursos disponibilizados por estes programas. Enquanto o PROGRESA, no México, começou em 1997 com aproximadamente 300 mil famílias beneficiárias; em 2013 alcançou 5,9 milhões de famílias, $25 \%$ destas vivem em comunidades indígenas. Já o Programa Bolsa Família (PBF) no Brasil começou em 2003 com quase 178 mil famílias e alcançou 13,8 milhões em 2016, quase um quarto da população total (Segura-Pérez, Graseda e Pérez-Escamilla, 2016).

Em outros países, o aumento no número de beneficiários tem sido menos intenso, mas ainda notável. Na Colômbia, por exemplo, o objetivo inicial do programa era de 400 mil famílias, mas expandiu-se para cobrir 1,5 milhão de famílias beneficiárias em 2007. Em termos de abrangência absoluta, os PTRCs de diferentes países variam entre 14 milhões de famílias brasileiras e 215 mil famílias chilenas. Em termos de amplitude relativa, variam de, aproximadamente, $40 \%$ da população (Equador) a cerca de 20\% (Brasil e México). Em termos de orçamento, os custos variam de cerca de $0,50 \%$ do Produto Interno Bruto (PIB) em países como Brasil, Equador e México a 0,08\% do PIB, no Chile. A generosidade dos benefícios varia de $20 \%$ do consumo médio das famílias, no México, a $4 \%$ em Honduras (Fiszbein, e Schady, 2009; Segura-Pérez, Graseda e Pérez-Escamilla, 2016).

No Brasil o Programa Bolsa Família (PBF) foi criado pelo Governo Federal em 2003, ao unificar os procedimentos de gestão e execução de várias ações de transferência de renda que atuavam de forma sobreposta, com baixa cobertura e com limitações administrativas, desde 1997. Após a fusão destes programas em outubro de 2003, a configuração do PBF permanece sem grandes alterações. Atualmente a gestão central do programa junto ao governo Federal está sob a coordenação do Ministério do Desenvolvimento Social (Oliveira e Daroit, 2020).

Quanto ao caráter, o programa é de proteção social, não contributivo, tal que as famílias destinatárias recebem transferências monetárias do Governo Federal, independentemente de sua participação no mercado de trabalho formal. É importante lembrar que, para $12 \%$ das famílias beneficiárias - aquelas que recebem apenas o benefício fixo -, o Bolsa Família é apenas um programa focalizado. Para as demais famílias, em situação de pobreza, o programa é tanto focalizado quanto condicionado, sendo que é necessário comprovar que os filhos frequentam a escola (Oliveira e Soares, 2012; Oliveira e Daroit, 2020).

Desde a sua criação, o programa atualizou os valores que definem pobreza e extrema pobreza. Em 2004, estes valores eram de até $\mathrm{R} \$ 50,00$ mensais per capita, para ser considerado de extrema pobreza e de $\mathrm{R} \$ 50,01$ a $\mathrm{R} \$ 100,00$ de renda mensal por pessoa para uma família ser considerada pobre. Em 2016, esses valores foram corrigidos para até $\mathrm{R} \$ \mathbf{8 5 , 0 0}$ por pessoa para qualificar como extrema pobreza e de $\mathrm{R} \$ 85,01$ a $\mathrm{R} \$ 170,00$ por pessoa, desde que tenha crianças ou adolescentes de 0 a 17 anos, para qualificar como pobre. 
Tabela 1- Benefícios variáveis e totais, mínimos e máximos do Programa Bolsa

\begin{tabular}{|c|c|c|c|c|}
\hline \multicolumn{5}{|c|}{ Família (R\$) - 2015} \\
\hline $\begin{array}{l}\text { Benefícios } \\
\text { variáveis }^{1} \\
\quad(\mathrm{R})\end{array}$ & $\begin{array}{c}\mathrm{BVVA}^{2} \\
(\mathrm{R} \$)\end{array}$ & $\begin{array}{c}\text { Total } \\
\text { Benefícios } \\
\text { Pobreza }^{3} \\
\text { (R\$) }\end{array}$ & $\begin{array}{l}\text { Benefício } \\
\text { Básico } \\
(R \$)\end{array}$ & $\begin{array}{c}\text { Total Benefícios } \\
\text { Extrema Pobreza } \\
(\mathrm{R} \$)\end{array}$ \\
\hline & & & 85,00 & 85,00 \\
\hline$x 1=39,00$ & & 39,00 & 85,00 & 124,00 \\
\hline$\times 5=195,00$ & & 195,00 & 85,00 & 280,00 \\
\hline$\times 5=195,00$ & $\times 2=92,00$ & 287,00 & 85,00 & 372,00 \\
\hline
\end{tabular}

(1) Benefício Variáveis crianças e adolescentes, gestantes e nutrizes - R\$ 39,00 - até 5 filhos por família. (2) Benefício variável vinculado ao adolescente - R\$46,00 - até 2 por família. (3) Renda per capita até $R \$ 85,00$. (4) Renda per capita entre $R \$ 85,00$ e $R \$ 170,00$. Fonte: Elaboração própria com dados do MDS, 2015.

A Tabela 1 detalha os benefícios variáveis e totais, mínimos e máximos que pode receber uma família em situação de extrema pobreza ou em situação de pobreza. Os beneficiários em situação de pobreza, coluna 3 na tabela, podem receber Benefícios Variáveis até o máximo de cinco benefícios e, no máximo, dois BVVA (Benefício Variável Vinculado ao Adolescente entre 16 e 17 anos de idade). Assim os beneficiários deste grupo podem receber de $\mathrm{R} \$$ 39,00 (um Benefício Variável de $R \$ 39,00$ ) até $R \$ 287,00$ (cinco BV de $R \$ 39,00$ mais dois BVVA de $R \$$ 46,00).

Os beneficiários em situação de extrema pobreza podem receber os dois tipos de benefícios. O denominado Benefício Básico de $\mathrm{R} \$ \mathbf{8 5 , 0 0}$ e os Benefícios Variáveis, de acordo ao número de crianças, adolescente, gestantes ou nutrizes na unidade familiar. Este último é limitado até um máximo de sete se tiver dois adolescentes entre 16 e 17 anos de idade. Neste caso os benefícios variam de $R \$$ 85,00 até $R \$ 372,00$, o que corresponde as colunas 4 e 5 da tabela 1 .

\subsection{Os Programas de Transferência de Renda Condicionada e o trabalho informal}

No livro "Buenas Intenciones, Malos Resultados", Levy (2010) apresenta um marco teórico mostrando de que forma as políticas sociais implementadas no México estimulam tanto trabalhadores como empresas a desenvolver atividades na informalidade. Basicamente, ele considera que existem dois setores de atividade quanto ao trabalho laboral, o formal e o informal. O setor formal consiste em empregos fornecidos pelas empresas, com todos os direitos trabalhistas. $O$ setor informal é aquele que não realiza o pagamento dos aportes ao sistema de seguridade social. Ao mesmo tempo, para proteger os setores que permanecem vulneráveis, o governo mexicano oferece benefícios de proteção social de forma gratuita aos trabalhadores do setor informal.

Assim, assegura Levy, o encarecimento dos salários do setor formal reduz a demanda por parte das empresas, nesse mercado, e os benefícios de proteção social estimulam a oferta de trabalho no setor informal (Levy, 2010).

Ahmad e Best (2012) apresentam um quadro teórico para a análise da política fiscal e de programas de benefícios sociais, em países com setor informal 
significativo. Especificamente, estudam os efeitos, no mercado laboral, de mão-deobra pouco qualificada, de mudanças dos benefícios sociais (nos mercados formal e informal), entre outras variáveis.

Um aumento na TMIG (Targeted Minimum Income Guarantee - Renda Mínima Garantida) provoca a diminuição da oferta laboral. Isso ocorre porque, embora o benefício TMIG seja direcionado para famílias sem trabalho, a implementação é imperfeita, já que os trabalhadores com empregos informais podem ainda receber o benefício. Isto torna o trabalho informal mais atraente e o trabalho formal menos atraente, diminuindo a oferta laboral formal. Isso significa que um alto nível de TMIG tornará mais atraente ser um trabalhador informal, aumentando a informalidade; já os empregadores formais terão que aumentar os salários formais para atrair trabalhadores, efetivamente taxando o emprego formal, reduzindo a produtividade e a eficiência.

No caso de aumento nos benefícios dos trabalhadores no setor formal, a oferta laboral aumenta, fazendo que o salário do setor formal diminua. Como a demanda relativa não é perfeitamente inelástica, a queda do salário não será suficiente para compensar o aumento do consumo decorrente do aumento do benefício formal. Assim, os trabalhadores formais estarão melhor colocados, tornando mais atraente aceitar um trabalho dentro da formalidade, aumentando a oferta, nesse mercado (Ahmad, et al., 2012).

Para Levy (2010), com o tempo, políticas que estimulam o setor informal podem provocar um ciclo vicioso prejudicial à produtividade. Como uma menor produtividade resulta em salários reais mais baixos, é compreensível que o governo tente melhorar o nível de vida dos trabalhadores com mais programas sociais, em particular, para os destinados ao setor informal. Isto, por sua vez, amplia ainda mais a brecha entre o custo do emprego formal e informal, implicando em mais empregados informais sem cobertura social.

Ao contrário de Levy, Cechini (2013) argumenta que existe uma relação positiva entre proteção social e emprego, de tal forma que os beneficiários do PTRC teriam mais oportunidades de buscarem melhores posições de trabalho e remuneração. $O$ autor também menciona que a proteção social equivale a um investimento em capacidades humanas, com benefícios em termos de produtividade do trabalho e coesão social.

Não mesma linha, Silva (2019) conclui que o PTRC não incentiva a informalidade e tampouco reduz a participação dos beneficiários no mercado de trabalho. Para a autora os beneficiários tendem a participar mais no mercado de trabalho quando comparado com os beneficiários na mesma faixa de renda.

\subsection{Evidências Empíricas}

A evidência empírica dos impactos de programas de transferência de renda condicionada, na escolha ocupacional do adulto beneficiário do programa, ainda está incipiente. Barbosa e Corseuil (2011) analisam estatísticas descritivas, a partir de dados da Pesquisa de Orçamentos Familiares (POF) de 2008 e 2009 do IBGE. Skoufias e Di Maro (2006), Ribas e Soares (2011) e Brauw et al. (2012) empregam alguma versão do estimador de diferenças em diferenças. A hipótese de identificação compartilhada, nesses trabalhos, portanto, é a de que as 
características não observáveis dos indivíduos, que interferem na seleção do programa, são fixas no tempo. Por outro lado, também, Barbosa e Corseuil (2014) adotam uma estratégia de identificação do efeito do PBF na escolha ocupacional, que procura isolar o real efeito do programa de diferenças em características não observáveis dos indivíduos. Veja na tabela 2 a seguir um resumo dos trabalhos de pesquisa em esta área.

Tabela 2 - Resumo de trabalhos focados em impactos do Programa Bolsa Família no mercado laboral informal

\begin{tabular}{|c|c|c|c|c|c|}
\hline Autores & Ano & Método & Dados & $\begin{array}{l}\text { Período } \\
\text { dos dados }\end{array}$ & Conclusões \\
\hline $\begin{array}{l}\text { Skoufias e } \\
\text { Di Maro }\end{array}$ & 2006 & $\begin{array}{l}\text { Diferencias em } \\
\text { diferencias }\end{array}$ & PROGRESA & $\begin{array}{l}1997 \text { a } \\
1999 \\
\text { Dados em } \\
\text { painel }\end{array}$ & $\begin{array}{l}\text { PTRC tem efeito sobre } \\
\text { a participação de } \\
\text { trabalhadores por } \\
\text { pouco tempo. }\end{array}$ \\
\hline $\begin{array}{l}\text { Barbosa e } \\
\text { Corseuil }\end{array}$ & 2011 & $\begin{array}{l}\text { Estatística } \\
\text { Comparativa }\end{array}$ & POF & $\begin{array}{l}2008 \mathrm{e} \\
2009\end{array}$ & $\begin{array}{l}\text { PBF altera a escolha } \\
\text { do tipo de trabalho } \\
\text { principal dos chefes } \\
\text { de famílias } \\
\text { beneficiárias }\end{array}$ \\
\hline $\begin{array}{l}\text { Ribas e } \\
\text { Soares }\end{array}$ & 2011 & $\begin{array}{l}\text { Generalized } \\
\text { Propensity Score }\end{array}$ & PNAD & $\begin{array}{l}\text { 2001, } 2004 \\
\text { e } 2006 \\
\text { Dados em } \\
\text { Painel }\end{array}$ & $\begin{array}{l}\text { Reduz a participação } \\
\text { da principal fonte de } \\
\text { renda do trabalho das } \\
\text { famílias no setor } \\
\text { formal nas áreas } \\
\text { metropolitanas }\end{array}$ \\
\hline $\begin{array}{l}\text { Brauw, et } \\
\text { al. }\end{array}$ & 2012 & $\begin{array}{l}\text { Propensity score } \\
\text { weighting }\end{array}$ & $\begin{array}{l}\text { Cadastro } \\
\text { Único e PBF }\end{array}$ & $\begin{array}{l}2005 \mathrm{e} \\
2009 \\
\text { Dados em } \\
\text { Painel }\end{array}$ & $\begin{array}{l}\text { Alguma evidência que } \\
\text { os homens têm } \\
\text { trabalhado mais horas } \\
\text { no setor informal. }\end{array}$ \\
\hline $\begin{array}{l}\text { Barbosa e } \\
\text { Corseuil }\end{array}$ & 2014 & $\begin{array}{l}\text { Regressão de } \\
\text { descont. Difusa }\end{array}$ & $\begin{array}{l}\text { PNAD Micro } \\
\text { dados }\end{array}$ & $\begin{array}{l}2006 \\
\text { Cross } \\
\text { Section }\end{array}$ & $\begin{array}{l}\text { O PBF não tem } \\
\text { impactos sobre a } \\
\text { escolha ocupacional } \\
\text { dos beneficiários } \\
\text { entre os postos } \\
\text { formais e informais. }\end{array}$ \\
\hline $\begin{array}{l}\text { Santos, et } \\
\text { al. }\end{array}$ & 2017 & $\begin{array}{l}\text { Modelo } \\
\text { semiparamétrico } \\
\text { de Cox }\end{array}$ & $\begin{array}{l}\text { RAIS, } \\
\text { CADúnico }\end{array}$ & 2011 & $\begin{array}{l}\text { O PBF aumenta a } \\
\text { probabilidade dos } \\
\text { beneficiários } \\
\text { permanecerem } \\
\text { empregados no setor } \\
\text { formal. }\end{array}$ \\
\hline $\begin{array}{l}\text { Correa } \\
\text { Junior, } \\
\text { Trevisan e } \\
\text { Mello }\end{array}$ & 2019 & $\begin{array}{l}\text { Modelo } \\
\text { econométrico: } \\
\text { dados em corte, } \\
\text { painel estático e } \\
\text { dinâmico }\end{array}$ & $\begin{array}{l}\text { CADúnico, } \\
\text { PNAD }\end{array}$ & $\begin{array}{l}2004 \text { a } \\
2013 \\
\text { Dados em } \\
\text { Painel }\end{array}$ & $\begin{array}{l}\text { O PBF aumenta o } \\
\text { número de pessoas no } \\
\text { mercado formal e os } \\
\text { salários recebidos. }\end{array}$ \\
\hline
\end{tabular}

Fonte: Organizado pelos autores.

O resultado de Skoufias e Di Maro (2006) para o México aponta um efeito sobre a participação de trabalhadores por conta própria, imediatamente após a 
implantação do programa Oportunidades. No entanto, esse efeito desaparece pouco tempo depois.

Barbosa e Corseuil (2011), no primeiro trabalho em que tratam do tema, utilizam estatísticas descritivas para tentar responder à pergunta se o PBF distorce a escolha ocupacional do chefe de domicílio. Eles utilizam a composição dos orçamentos domésticos das famílias e as características demográficas dos domicílios da Pesquisa de Orçamentos Familiares (POF) de 2008 e 2009 pelo IBGE. A amostra é dividida nos seguintes grupos: "não elegíveis - quase elegíveis" e, "elegíveis - quase não elegíveis". A delimitação desses grupos específicos é o aspecto central da metodologia. Nesse sentido, os grupos foram delimitados para contrastar as decisões de domicílios não elegíveis, porém "quase elegíveis" com as decisões tomadas em domicílios elegíveis, porém "quase inelegíveis". A hipótese fundamental, que permite usar tal comparação como uma medida do impacto do PBF na escolha do tipo de ocupação no principal trabalho dos chefes de famílias beneficiárias do programa, é a de que todos os demais fatores que afetam tal escolha o fazem da mesma forma, num grupo ou no outro. Sob esta hipótese, Barbosa e Corseuil (2011) concluem que o PBF altera, de fato, a escolha do tipo de trabalho principal dos chefes de famílias beneficiárias.

Ribas e Soares (2011) utilizam uma abordagem baseada em GPS (Generalized Propensity Score) para estimar o impacto do PBF sobre a oferta de mão-de-obra, no Brasil. Extraem os dados da Pesquisa Nacional de Amostras por Domicílio (PNAD) de 2004 e 2006. Eles defendem a hipótese que, quando o programa vai de áreas isoladas para grandes cidades e todos ficam informados sobre suas regras, os impactos podem ser diferentes. O estudo verifica que o benefício efetivamente amplia a participação dos trabalhadores adicionais das famílias nas áreas rurais. Por outro lado, reduz a participação da principal fonte de renda do trabalho das famílias no setor formal, nas áreas metropolitanas (Ribas, et al., 2011).

A segunda rodada da AIBF (Avaliação de Impacto do Programa Bolsa Família) utiliza dados do Cadastro Único e do Programa Bolsa Família em 2005 e 2009 e a metodologia para esta avaliação é a propensity score weighting. Brauw, et al. (2012) concluem que há alguma evidência de que, nos domicílios participantes, os homens têm trabalhado menos horas por semana no setor formal e mais horas no setor informal.

Barbosa e Corseuil (2014) aplicam o método de regressão de descontinuidade difusa, explorando a descontinuidade presente nas regras de elegibilidade do programa. Diversos trabalhos que procuram aferir os benefícios do PBF exploraram a condição de elegibilidade baseada na Renda Domiciliar per capita (RDPC). Dado que o rendimento de uma ocupação informal pode ser ocultado dos agentes responsáveis pelo PBF, há espaço para a auto seleção; e a probabilidade de um indivíduo entrar no programa depende de características não observáveis. Por este motivo, os autores optaram por explorar a condição de elegibilidade baseada na idade do filho mais novo, por ser uma variável mais difícil de ser manipulada. A análise é feita com base nos microdados da Pesquisa Nacional por Amostra de Domicílios (PNAD) de 2006, do IBGE. Os resultados encontrados sugerem que o programa não tem impactos sobre a escolha ocupacional dos beneficiários entre os postos formais e informais. 
Já Santos et al (2017) analisam os efeitos do PBF na duração dos vínculos empregatícios no mercado de trabalho formal comparando indivíduos de baixa renda, beneficiários com os não beneficiários do programa. Para isto utilizam de um modelo semiparamétrico de sobrevivência (Cox), estimando a probabilidade de desligamento dos beneficiários versus não beneficiários do PBF. A partir dos dados da RAIS, CADúnico e da folha de pagamento dos beneficiários do PBF com informações de 2011, é estimado que os beneficiários do programa possuem maiores chances de permanecerem no mercado formal de trabalho do que os não beneficiários. Os autores destacam que o risco de desligamento do emprego para os beneficiários do PBF é de 7\% a 10\% menor do que o de não beneficiários.

Por fim, o estudo de Correa Junior, Trevisan e Mello (2019) analisa a relação entre PBF e a participação dos trabalhadores no mercado formal brasileiro. $O$ estudo utiliza três métodos econométricos para análise dos dados: econometria com dados em corte, modelos em painel estático e modelos em painel dinâmico. Os atores defendem que o PBF possui benefícios que vão além de um programa de benefícios social para combater a pobreza e melhorar a saúde e educação das famílias. Os autores consideram que o PBF também possui efeitos positivos sobre o mercado de trabalho formal, como o aumento no número de pessoas empregadas e o incremento nos salários recebidos.

Com base nos três primeiros estudos brasileiros, estes encontraram evidências de que o PBF estimulou os beneficiários, as principais fontes de renda das famílias, a escolherem empregos no mercado laboral informal, assim, sendo invisíveis aos gestores do programa, seguindo com o recebimento do benefício, ainda que não se enquadrassem nas especificações para recebê-lo. O trabalho de Barbosa e Corseuil (2014) não confirma esta tendência, mostrando que o PBF não tem impacto sobre a escolha ocupacional entre mercado de trabalho formal e informal. Por outro lado, Santos, et al (2017) e Correa Junior, Trevisan e Mello (2019) mostram evidências que o PBF tem efeitos positivos sobre a permanência no mercado formal por parte da população de baixa renda beneficiada pelo programa.

\section{Metodologia}

A metodologia ${ }^{1}$ proposta utiliza-se de modelos multivariados para avaliar as relações de curto e longo prazo entre as variáveis. Inicialmente é analisado o comportamento das variáveis quanto a estacionaridade e, sendo as variáveis não estacionárias, verifica-se a existência de cointegração ou relação de longo prazo entre as variáveis.

Com o propósito de estimar modelos que combinam propriedades de curto e longo prazo, utiliza-se do Vetor de Correção de Erros (VEC) para estimar a relação entre as variáveis ao longo do tempo. O VEC pode ser apresentado de forma simplificada em termos de três variáveis, $Y_{t}, X_{t}$ e $W_{t}$, em que $k=1$ (sendo $k$ o número de defasagens), o modelo é o seguinte (equação 1):

\footnotetext{
${ }^{1}$ Esta seção utilizou como referência a (Dimitrios Asteriou, 2006), capítulo 17.
} 


$$
\left(\begin{array}{l}
\Delta Y_{t} \\
\Delta X_{t} \\
\Delta W_{t}
\end{array}\right)=\Gamma_{1}\left(\begin{array}{c}
\Delta Y_{t-1} \\
\Delta X_{t-1} \\
\Delta W_{t-1}
\end{array}\right)+\Pi\left(\begin{array}{c}
Y_{t-1} \\
X_{t-1} \\
W_{t-1}
\end{array}\right)+\varepsilon_{t}
$$

$\mathrm{Na}$ equação 2 , a matriz $\Pi$ contém a informação referente as relações de longo prazo. Ao mesmo tempo pode ser decomposto em $\Pi=\alpha \theta^{\prime}$, aonde $\alpha$, inclui os coeficientes de velocidade de ajuste de equilíbrio, enquanto $b^{\prime}$ é a matriz de coeficientes de longo prazo. Que pode expressar-se da seguinte forma:

$$
\left(\begin{array}{l}
\Delta Y_{t} \\
\Delta X_{t} \\
\Delta W_{t}
\end{array}\right)=\Gamma_{1}\left(\begin{array}{l}
\Delta Y_{t-1} \\
\Delta X_{t-1} \\
\Delta W_{t-1}
\end{array}\right)+\left(\begin{array}{ll}
a_{11} & a_{12} \\
a_{21} & a_{22} \\
a_{31} & a_{32}
\end{array}\right)\left(\begin{array}{lll}
\beta_{11} & \beta_{12} & \beta_{13} \\
\beta_{21} & \beta_{22} & \beta_{23}
\end{array}\right)\left(\begin{array}{c}
Y_{t-1} \\
X_{t-1} \\
W_{t-1}
\end{array}\right)+\varepsilon_{t}
$$

O VEC da primeira equação, por exemplo para $\Delta Y_{t}$, resulta em:

$$
\Pi_{1} Z_{t-1}=\left(\left[a_{11} \beta_{11}+a_{12} \beta_{12}\right]\left[a_{11} \beta_{21}+a_{12} \beta_{22}\right]\left[a_{11} \beta_{31}+a_{12} \beta_{32}\right]\right)\left(\begin{array}{c}
Y_{t-1} \\
X_{t-1} \\
W_{t-1}
\end{array}\right)
$$

onde $\Pi_{1}$ é a primeira fila da matriz $\Pi$.

Pode-se reorganizar a equação 3 da seguinte forma:

$$
\Pi_{1} Z_{t-1}=a_{11}\left(\beta_{11} Y_{t-1}+\beta_{21} X_{t-1}+\beta_{31} W_{t-1}\right)+a_{12}\left(\beta_{12} Y_{t-1}+\beta_{22} X_{t-1}+(4)_{32} W_{t-1}\right)
$$

onde se observam os dois vetores de cointegração com seus respectivos termos de ajustamento $a_{11}$ e $a_{12}$.

O VEC tem várias vantagens: em primeiro lugar, mede a correção do desequilíbrio do período prévio, que é uma implicação econômica interessante. Em segundo lugar, resolve o problema de regressões espúrias. A terceira vantagem do VEC é a facilidade com que ele pode se encaixar na abordagem "do geral ao específico" para a modelagem econométrica, que de fato é a busca do modelo mais parcimonioso.

É possível que o ponto mais importante seja o fato de que o resíduo é uma variável estacionária e, por este motivo, o VEC tem implicâncias importantes: o fato de que as variáveis são cointegradas implica que existe um processo de ajuste, ou correção ao longo do período analisado.

\subsection{Dados}

Os dados agregados, mensais, deste estudo se estendem pelo período de março 2004 até fevereiro de 2016, para as seis regiões metropolitanas incluídas na Pesquisa Mensal de Emprego - PME - do IBGE. Durante esse período conviveram a PME e o Programa Bolsa Família, já que a PME foi desativada pelo IBGE a partir de março de 2016. As regiões metropolitanas incluídas na pesquisa são: Recife (PE), Salvador (BA), Belo Horizonte (MG), Rio de Janeiro (RJ), São Paulo (SP) e Porto Alegre (RS). A tabela 3 mostra a quantidade de municípios existentes em cada região metropolitana e a população de cada uma. As seis regiões metropolitanas que compõem a PME abrangem um total de 51,6 milhões de habitantes em 143 municípios. 
Tabela 1 - Resumo das características das regiões metropolitanas que compõem o

\begin{tabular}{|c|c|c|}
\hline \multicolumn{3}{|c|}{ PME - IBGE 2016} \\
\hline $\begin{array}{l}\text { Região } \\
\text { Metropolitana }\end{array}$ & $\begin{array}{c}\text { Quantidade de } \\
\text { Municípios }\end{array}$ & População \\
\hline Belo Horizonte (MG) & 31 & 5.873 .841 \\
\hline Porto Alegre (RS) & 30 & 4.276 .475 \\
\hline Recife (PE) & 13 & 3.940 .456 \\
\hline Rio de Janeiro (RJ) & 20 & 12.330 .186 \\
\hline Salvador (BA) & 10 & 3.984 .583 \\
\hline São Paulo (SP) & 39 & 21.242 .939 \\
\hline Total & 143 & 51.648 .480 \\
\hline
\end{tabular}

Fonte: IBGE, Diretoria de Pesquisas - DPE, Coordenação de População e Indicadores Sociais - COPIS, 2016.

Considerando estas regiões metropolitanas, o modelo analisa a relação entre o mercado laboral informal e o número de pessoas desempregadas, a produção industrial e os benefícios do PBF. Conforme a tabela 4, os dados a respeito dos trabalhadores sem carteira assinada, trabalhadores por conta própria e trabalhadores sem emprego são extraídos da PME - IBGE. O número de trabalhadores empregados por conta própria e o de trabalhadores empregados sem carteira assinada, somadas, constituem o mercado laboral informal. O valor médio de benefício do PBF é calculado a partir de dados fornecidos pelo Ministério de Desenvolvimento Social e Combate à Fome, e deflacionado a valores de fevereiro de 2016 pelo IPCA, extraído do IBGE. O índice de produção industrial foi obtido na página Banco de Dados Séries Estatísticas e Séries Históricas do IBGE (em índice de base fixa mensal sem ajuste sazonal da produção industrial - Atividades Industriais - Brasil e Estados selecionados). Todas as variáveis foram dessazonalizadas, utilizando-se de dummies mensais.

Tabela 2 - Descrição e fonte das variáveis

\begin{tabular}{lcl}
\hline \multicolumn{1}{c}{ Descrição } & Variável & \multicolumn{1}{c}{ Fonte } \\
\hline $\begin{array}{l}\text { Mercado Laboral Informal: soma do } \\
\text { número de pessoas ocupadas sem carteira } \\
\text { assinada e o número de pessoas por conta } \\
\text { própria. }\end{array}$ & MLI & $\begin{array}{l}\text { Pesquisa Mensal de Emprego - } \\
\text { IBGE }\end{array}$ \\
$\begin{array}{l}\text { Benefício médio por beneficiário do } \\
\text { Programa Bolsa Família. }\end{array}$ & BFD & $\begin{array}{l}\text { Min. de Desenvolvimento } \\
\text { Social e Combate à Fome }\end{array}$ \\
$\begin{array}{l}\text { Índice de Produção Industrial } \\
\text { Número de pessoas desempregadas }\end{array}$ & PIN & $\begin{array}{l}\text { IBGE } \\
\text { DES }\end{array}$ \\
\hline
\end{tabular}

Fonte: Organizado pelos autores.

Na próxima seção, são apresentados os resultados e, posteriormente, as devidas conclusões. 


\section{Resultados}

Nesta seção inicialmente são apresentadas as estatísticas descritivas das variáveis e o comportamento delas ao longo do período analisado. Na sequência são realizados os testes de raiz unitária e o teste de cointegração, estimados o VEC, a causalidade de Granger e a função impulso resposta.

$\mathrm{Na}$ Tabela 5 podem ser observadas as estatísticas descritivas. Nesta tabela destaca-se a razão entre os valores máximos e mínimos. Efetivamente três variáveis mostram uma amplitude significativa, especificamente, os valores referentes ao benefício médio do Programa Bolsa Família por beneficiário $(1,7)$, o Índice de Produção Industrial $(1,52)$ e o número de pessoas desempregadas $(2,67)$. A variável que mostra a menor amplitude entre valor máximo e mínimo é "pessoas ocupadas no Mercado Laboral Informal (MLI)", neste caso o máximo é 1,16 vezes o valor mínimo.

Tabela 3 - Estatísticas Descritivas das variáveis utilizadas

(MLI, BFD, PIN e DES)

\begin{tabular}{l|r|r|r|r|r|r}
\hline Variável & Mínimo & \multicolumn{1}{|c|}{ Máximo } & Max/Min & Média & D. Padrão & \% Var. \\
\hline MLI & $7.348,0$ & $8.549,0$ & 1,16 & $8.058,5$ & 310,1 & $-9,0 \%$ \\
BFD & 101,5 & 172,7 & 1,70 & 133,1 & 21,6 & $4,1 \%$ \\
PIN & 74,0 & 112,6 & 1,52 & 95,7 & 8,9 & $-1,3 \%$ \\
DES & $1.073,4$ & $2.871,0$ & 2,67 & $1.799,2$ & 420,7 & $-19,2 \%$ \\
\hline
\end{tabular}

Fonte: Organizado pelos autores.

Nas figuras 1, 2 e 3 se observam os gráficos das quatro séries históricas das variáveis do modelo. Na primeira figura se observa que o número de pessoas ocupadas, no mercado informal, permaneceu mais estável quando comparada com as outras séries. A informalidade diminuiu $9 \%$ no período, quando se compara a variação ponta a ponta. A série também mostra certa volatilidade entre 2004 e 2010. A partir de este ponto, a série fica mais estável com tendência à baixa.



Figura 1 - Pessoas Ocupadas no Mercado Informal de 2004 a 2016 Fonte: PME-IBGE, 2016.

O benefício por beneficiário (Figura 2), mostra uma diminuição nos primeiros anos, até o começo de 2006 e, daí em diante, um constante aumento até setembro de 2014, quando reverte para uma tendência decrescente. Em ambos os casos a 
diminuição foi consequência de aumentos no número de beneficiários e não na diminuição de repasses.

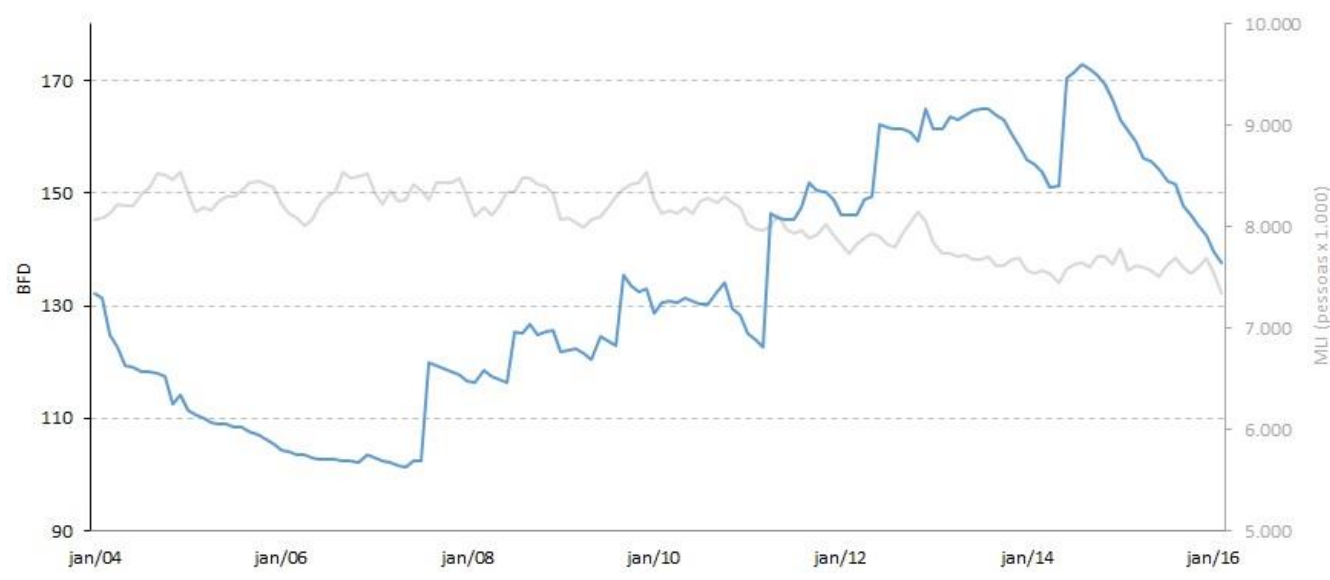

Figura 2 - Benefício por Família Beneficiária e Mercado Laboral Informal - 2004-16 Fonte: Min. de Desenvolvimento Social e Combate à Fome, 2016.

Por outro lado, observando as duas séries na Figura 2, se o PBF estimula a informalidade, na medida em que aumenta o benefício médio, deveria também aumentar a informalidade dos beneficiários, em cinza na figura. $O$ fato de haver aumentos dos benefícios médios, a simples vista na figura, não demonstra ter um efeito significativo no mercado laboral informal, deixando enfraquecida a ideia do "efeito informalidade" do PBF. Este resultado mostra exatamente esta ideia, ainda que o PBF estivesse estimulando a informalidade dos beneficiários, o tamanho do mercado laboral informal é tão importante que existem muitas outras variáveis nele atuando.

O número de pessoas desempregadas apresenta uma clara tendência decrescente na Figura 3. Esta figura mostra uma grande diferença entre os valores mínimo e máximo, sendo o segundo 2,7 vezes maior que o primeiro. A partir de 2014, a tendência é fortemente revertida até o final da série, refletindo os efeitos da crise nacional iniciada naquele ano. Outro detalhe que se deve destacar é a sazonalidade presente na série. Assim, pode-se observar diminuições dos índices cada final de ano. 


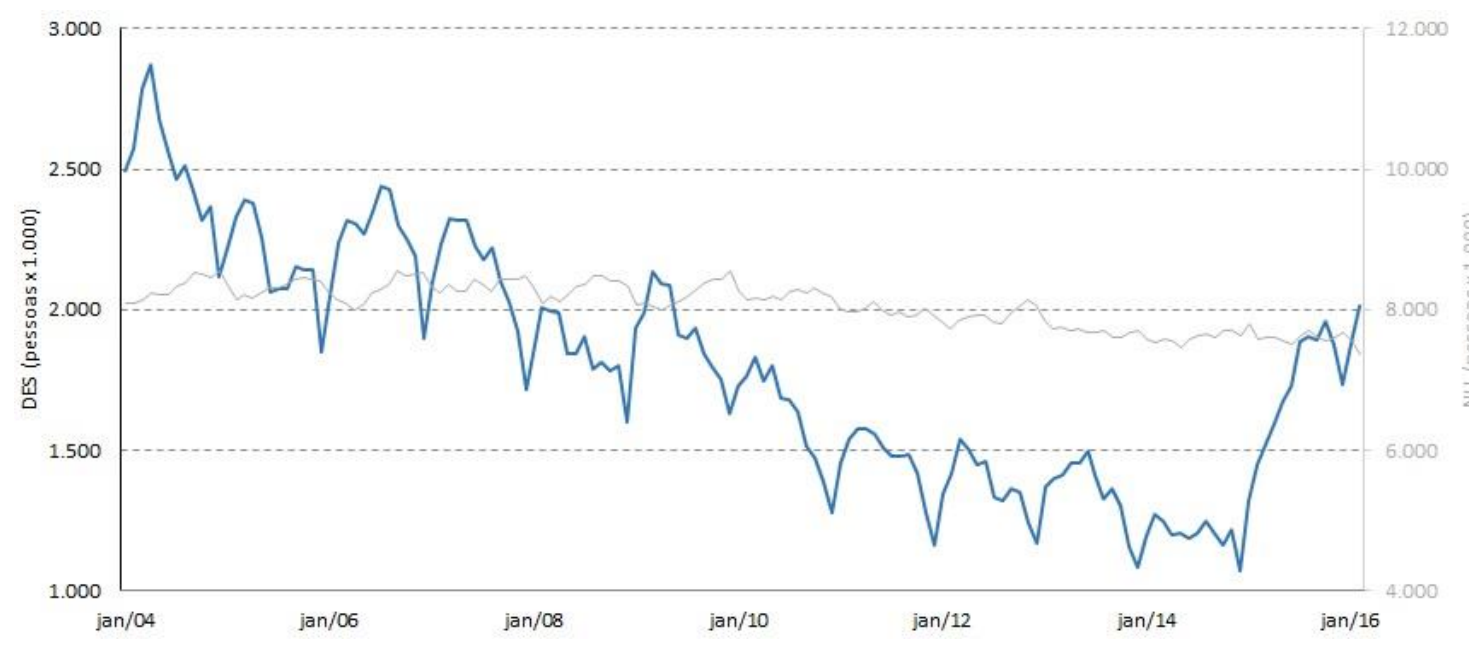

Figura 3 - Número de pessoas desempregadas e pessoas ocupadas na informalidade. Fonte: PME-IBGE, 2016.

Um detalhe interessante é observado quando se comparam as séries na Figura 3. É razoável supor que a diminuição no número de desempregados segue a mesma tendência do número de pessoas ocupadas na informalidade. Mas não é isso que se observa, se por um lado o desemprego efetivamente mostra uma diminuição importante entre 2004 até final de 2014; no mesmo período, o mercado laboral informal permanece relativamente estável.

A última variável que compõe o trabalho é um indicador de atividade. Esta série objetiva refletir uma atividade que interaja, especificamente, com potenciais trabalhadores elegíveis ao programa de forma mais próxima. Para tal fim foi escolhido o índice de Produção Industrial, que capta melhor que o PIB total a faixa socioeconômica, objeto do PBF. O Índice de Produção Industrial é obtido do IBGE em nível nacional. Na Figura 4 se observa que a série sofre fortes variações sazonais ao fim de cada ano. O crescimento, a partir de 2004, é interrompido em 2008 pela crise internacional. A partir desse período, o índice se mostra estável até 2014, quando começa a decrescer, em razão da crise nacional. 




Figura 4 - Índice de Produção Industrial e o Mercado Laboral Informal Fonte: IBGE, 2016.

No caso da comparação do Índice de Produção Industrial e o Mercado Laboral Informal se observa que os aumentos da produção industrial presentes entre 2004 e 2008 não são acompanhados em mesma intensidade pelo mercado laboral informal. Na medida em que a Produção Industrial se mantém estável entre 2009 e 2015, o Mercado Laboral Informal apresenta uma tendência suave e decrescente.

\subsection{Discussão dos resultados econométricos}

Inicialmente, os dados são dessazonalizados e é verificada a existência de quebra estrutural das séries utilizando o teste de Chow. Na sequência são realizados os testes de raiz unitária com quebra estrutural, estimação do VAR, teste de Casualidade de Granger e a análise Impulso-Resposta.

Na primeira parte da análise dos resultados foi realizado o teste proposto de (por?) Dickey-Fuller, que considera a possibilidade de quebra estrutural para dois modelos: a) modelo de outlier aditivo (OA), que captura mudanças abruptas nas séries; b) modelo de outlier inovacional (OI), que identifica mudanças graduais na média das séries.

A Tabela 6 apresenta os resultados dos testes de ADF com quebra estrutural. No modelo de outlier aditivo a série Desemprego (DES) é estacionária (I(0)) e as outras três séries são I(1). Para o modelo outlier inovacional, a única série estacionária é Mercado Laboral Informal (MLI), assim as outras três são I(1).

Tabela 4 - Teste de Raiz Unitária (ADF) com quebra estrutural

\begin{tabular}{cccccccc}
\cline { 2 - 7 } Série & \multicolumn{3}{c|}{ ADF (AO) } & \multicolumn{3}{c}{ ADF (OI) } \\
& $\begin{array}{c}\text { Estatística } \\
\text { variável em } \\
\text { nível }\end{array}$ & $\begin{array}{c}\text { Ordem de } \\
\text { Integração }\end{array}$ & $\begin{array}{c}\text { Ponto } \\
\text { de } \\
\text { quebra }\end{array}$ & $\begin{array}{c}\text { Estatística } \\
\text { variável em } \\
\text { nível }\end{array}$ & $\begin{array}{c}\text { Ordem de } \\
\text { Integração }\end{array}$ & $\begin{array}{c}\text { Ponto de } \\
\text { quebra }\end{array}$ \\
\hline \multirow{2}{*}{ MLI } & $\begin{array}{c}-4,767 \\
(0,065)\end{array}$ & $\mathrm{I}(1)$ & 2006.2 & $\begin{array}{c}-4.995 \\
(0,034)\end{array}$ & $\mathrm{I}(0)$ & 2006.05
\end{tabular}




\begin{tabular}{|c|c|c|c|c|c|c|}
\hline BFD & $\begin{array}{c}-3,993 \\
(0,376)\end{array}$ & $\mathrm{I}(1)$ & 2015.03 & $\begin{array}{l}-4,009 \\
(0,366)\end{array}$ & $\mathrm{I}(1)$ & 2015.03 \\
\hline DES & $\begin{array}{l}-5.325 \\
(0,011)\end{array}$ & $\mathrm{I}(0)$ & 2014.4 & $\begin{array}{l}-4,466 \\
(0,143)\end{array}$ & $\mathrm{I}(1)$ & 2015.04 \\
\hline PIN & $\begin{array}{c}-4,221 \\
(0,247)\end{array}$ & $\mathrm{I}(1)$ & 2013.12 & $\begin{array}{c}-3,211 \\
(0,841)\end{array}$ & $\mathrm{I}(1)$ & 2014.10 \\
\hline
\end{tabular}

Fonte: resultados de pesquisa.

Como há séries que não são estacionárias (I(1)), o próximo passo é verificar se as séries são cointegradas. Inicialmente é avaliado o número ideal de defasagens para a estimação do VAR, por meio dos critérios de informação de Akaike, Schwarz e Hannan-Quinn. Conforme a Tabela 7 uma defasagem é apropriada para as variáveis do modelo.

Tabela 5-Resultados quanto ao número ótimo de defasagens do VAR

\begin{tabular}{cccc}
\hline \hline Lag & AIC & SC & HQ \\
\hline \hline 0 & 40.26353 & 40.35003 & 40.29868 \\
1 & $32.73924^{*}$ & $33.17176^{*}$ & $32.91500^{*}$ \\
2 & 32.81839 & 33.59692 & 33.13476 \\
3 & 32.90984 & 34.03438 & 33.36682 \\
4 & 32.92006 & 34.39061 & 33.51764 \\
5 & 33.03020 & 34.84675 & 33.76839 \\
\hline \hline
\end{tabular}

Fonte: resultados de pesquisa.

Se existem séries integradas de ordem 1, pode existir equilíbrio de longo prazo entre elas, ou, em outras palavras, podem ser cointegradas. Assim, depois de definir o número ideal de defasagens, realiza-se o Teste de Cointegração de Johansen. Este teste verifica o número de vetores cointegrantes que podem ser considerados estatisticamente significativos no modelo proposto. O resultado do Teste de Cointegração de Johansen, conforme a Tabela 8, indica que as séries têm, no máximo, dois vetores de cointegração a um nível de significância de $5 \%$, indicando a existência de uma relação de equilíbrio de longo prazo entre as séries.

Tabela 6 - Teste de Cointegração de Johansen

\begin{tabular}{ccccc}
\hline $\begin{array}{c}\text { Hypothesized } \\
\text { No. of CE (s) }\end{array}$ & Eigenvalue & $\begin{array}{c}\text { Trace } \\
\text { Statistic }\end{array}$ & $\begin{array}{c}\text { 0.05Critical } \\
\text { Value }\end{array}$ & Prob.** \\
\hline \hline None * & 0.151113 & 60.45524 & 54.07904 & 0.0121 \\
At most 1 * & $\mathbf{0 . 1 2 7 1 6 8}$ & 36.86379 & 35.19275 & $\mathbf{0 . 0 3 2 7}$ \\
At most 2 & 0.082333 & 17.27803 & 20.26184 & 0.1225 \\
At most 3 & 0.033492 & 4.905442 & 9.164546 & 0.2939 \\
\hline \hline
\end{tabular}

Fonte: resultados de pesquisa.

Assim, em vista que os testes e critérios de seleção definiram que as quatro séries, dessazonalizadas, têm quebra estrutural; algumas séries não são estacionárias, deve-se aplicar uma defasagem na regressão. Ao mesmo tempo, o 
teste de cointegração de Johansen indica que as séries são cointegradas e que devem ser estimadas com duas equações cointegradas, e a segunda especificação algébrica, com intercepto e sem tendência na equação de cointegração e sem intercepto no VAR. Como as variáveis são não estacionárias e cointegradas, pode-se aplicar o VEC (Vetor de Correção de Erros) para estimar a regressão.

Os resultados para o modelo VEC são apresentados na Tabela 9. Os coeficientes associados as variáveis em primeira diferença defasados correspondem as relações de curto prazo entre as variáveis. Os valores entre parênteses são as estatísticas t.

Tabela 7 - Vetor Corretor de Erros - Modelos de Curto Prazo

\begin{tabular}{ccccccc} 
& $\Delta \mathrm{MLI}_{\mathrm{t}-1}$ & $\Delta \mathrm{PIN}_{\mathrm{t}-1}$ & $\Delta \mathrm{BFD}_{\mathrm{t}-1}$ & $\Delta \mathrm{DES}_{\mathrm{t}-1}$ & $\mathrm{VC} 1$ & $\mathrm{VC} 2$ \\
\hline$\Delta \mathrm{MLI}$ & $-0,133$ & 0,857 & 1,278 & 0,099 & $-0,034$ & 2,106 \\
$\mathrm{t}$ & {$[-1,572]$} & {$[0,355]$} & {$[0,714]$} & {$[1,035]$} & {$[-1,174]$} & {$[1,060]$} \\
& & & & & & \\
$\Delta$ PIN $_{\mathrm{t}}$ & 0,001 & $-0,207$ & 0,086 & $-0,006$ & 0,003 & $-0,212$ \\
& {$[0,246]$} & {$[-2,440]$} & {$[1,371]$} & {$[-1,839]$} & {$[2,880]$} & {$[-3,047]$} \\
$\Delta \mathrm{BFD}$ & $-0,004$ & $-0,038$ & $-0,078$ & 0,003 & 0,001 & 0,019 \\
$\mathrm{t}$ & {$[-1,065]$} & {$[-0,349]$} & {$[-0,975]$} & {$[0,580]$} & {$[0,524]$} & {$[0,216]$} \\
& & & & & & \\
$\Delta \mathrm{DES}$ & $-0,006$ & $-0,723$ & $-3,003$ & 0,000 & 0,068 & $-4,513$ \\
$\mathrm{t}$ & {$[-0,077]$} & {$[-0,333]$} & {$[-0,333]$} & {$[0,002]$} & {$[2,588]$} & {$[-2,525]$} \\
\hline
\end{tabular}

Fonte: resultados de pesquisa.

A partir da estimação do VEC podem-se também observar os dois vetores de cointegração, ou de equilíbrio de longo prazo, VC1 e VC2, com os "t estatísticos" em parênteses, na Tabela 10.

Tabela 8 - Vetor Corretor de Erros - Modelos de Longo Prazo

\begin{tabular}{|c|c|c|c|c|c|c|}
\hline & & $\mathrm{MLI}_{\mathrm{t}-1}$ & $\mathrm{PIN}_{\mathrm{t}-1}$ & $\mathrm{BFD}_{\mathrm{t}-1}$ & $\mathrm{DES}_{\mathrm{t}-1}$ & $C$ \\
\hline \multirow[t]{2}{*}{ VC1 } & $\Rightarrow$ & 1,000 & 0,000 & $-99,786$ & $-6,538$ & 22,564 \\
\hline & & & & {$[-3,969]^{*}$} & {$[-4,973]^{*}$} & {$[0,081]$} \\
\hline \multirow[t]{2}{*}{$\mathrm{VC2}$} & $\Rightarrow$ & 0,000 & 1,000 & $-1,490$ & $-0,077$ & 0,565 \\
\hline & & & & {$[-4,003]^{*}$} & {$[-3,880]^{*}$} & {$[0,137]$} \\
\hline
\end{tabular}

*Coeficientes significativos ao $1 \%\left(t_{c}=2,326\right)$.

Fonte: resultados de pesquisa. 
A equação de equilíbrio de longo prazo mais apropriada é a VC1. Pela definição de cointegração, a equação representa uma relação de equilíbrio no longo prazo. Assim, pode-se isolar a variável representativa do mercado laboral informal:

$$
M L I_{t-1}=99,79 B F D_{t-1}+6,54 D E S_{t-1}-22,56
$$

A equação indica que, no longo prazo, o Programa Bolsa Família (BFD) tem uma relação positiva e significativa estatisticamente, com o Mercado Laboral Informal (MLI), implicando que, ao aumentar o valor médio do benefício, aumenta o número de pessoas no mercado laboral informal. A cada um Real de aumento do benefício médio, houve um aumento cem pessoas no mercado laboral informal, mantendo constante o desemprego (DES). Deve-se destacar, também, que, no longo prazo, o desemprego também tem uma relação positiva e estatisticamente significativa com o mercado laboral informal, o que implica que aumentos no número de desempregados resultam em maior quantidade de pessoas no mercado informal, o que está alinhado com a teoria econômica. As estatísticas em parênteses, na Tabela 10, indicam que todos os coeficientes estimados pelo modelo são estatisticamente significativos.

Outra informação importante consiste na relação de curto prazo entre as variáveis a partir da primeira equação da Tabela 9:

$\Delta M L I_{t}=-0,133 \Delta M L I_{t-1}+0,857 \Delta P I N_{t-1}+1,278 \Delta B F D_{t-1}+0,099 \Delta D E S_{t-1}-0,034 V C 1+$ $2,106 \mathrm{VC2}$

Observa-se, na equação de equilíbrio de curto prazo, e de acordo ao coeficiente de VC1, que 3\% do desequilíbrio do período anterior se ajusta no período atual. Por exemplo, quando o mercado laboral informal excede seus níveis de equilíbrio de longo prazo, se tem a seguinte relação:

$$
M L I_{t-1}>99,79 B F D_{t-1}+6,54 D E S_{t-1}-22,56 \Longrightarrow V C_{1}>0
$$

Assim, em níveis superiores aos de equilíbrio (VC1>0), no mercado laboral, é fornecido um valor positivo da equação de cointegração e se reflete como uma diminuição na relação de curto prazo pelo coeficiente negativo de 0,03. Isso leva a relação de curto prazo ao equilíbrio novamente.

Uma outra forma de analisar a possibilidade de causalidade entre variáveis é realizar o teste de Causalidade de Granger. A causalidade de Granger baseia-se na ideia de que se $X$ causa $Y$, o conhecimento de valores passados de $X$ permitem melhores previsões de $Y$. Trata-se, pois, de um teste de precedência temporal e não de causalidade no sentido de uma relação de causa e efeito. A Tabela 11 mostra os resultados do teste.

Tabela 9- Teste Causalidade de Granger

$\left.\begin{array}{cc}\hline \text { Causalidade } & \text { Interpretação } \\ B F D \rightarrow M L I & \text { BFD influencia o } M L I \\ {[0,003]} & M L I \text { não influencia o BFD }\end{array}\right] \begin{gathered}\text { BFD tem causalidade } \\ \text { de Granger com o } M L I\end{gathered}$




$$
[0,845]
$$

$\mathrm{DES} \rightarrow \mathrm{MLI}$

$[0,009]$

$M L I \rightarrow D E S$

$[0,228]$

$\mathrm{DES} \rightarrow \mathrm{BFD}$

$[0,000]$

$\mathrm{BFD} \rightarrow \mathrm{DES}$

$[0,855]$
DES influencia o $M L I$

$M L I$ não influencia o DES

DES influencia o BFD

BFD não influencia o DES
DES tem causalidade

de Granger com o MLI

DES tem causalidade de Granger com o BFD

Fonte: resultados de pesquisa.

Os resultados apresentados na Tabela 11 mostram que o Programa Bolsa Família causa mudanças no mercado laboral informal, todavia o inverso não aparece como verdadeiro. Outra relação causal importante observada é o efeito causal e positivo da taxa de desemprego sobre o número de trabalhadores no mercado informal no Brasil. Por fim, mostrou-se também significativa a relação causal no sentido de Granger do número de desempregados em relação ao número de pessoas contempladas com o Programa Bolsa Família, destacando o efeito contra cíclico deste programa no Brasil nos períodos de elevada taxa de desemprego.

Embora exista uma relação positiva e causal entre o número de beneficiários com Bolsa Família e o número de trabalhadores no mercado Informal, ao investigarse a relação de impulso e resposta (Figura 5) entre estas duas variáveis o efeito do Programa de Bolsa Família sobre o mercado informal brasileiro mostrou-se muito pequeno, embora com um efeito permanente no longo prazo.

\section{Response to Cholesky One S.D. Innovations}



Figura 5 - Função Impulso-Resposta Beneficiários Bolsa Família sobre o

Mercado Laboral Informal Brasileiro.

Desta forma, o presente trabalho não identificou evidências que o Programa Bolsa Família tenha contribuído para aumentar significativamente o mercado laboral informal brasileiro no período analisado. Ainda com relação aos resultados quanto a interação entre as variáveis do trabalho e a função impulso-resposta, cabe destacar que as variáveis analisadas conjuntamente não tiveram um efeito relevante 
em termos de magnitude, o que também pode ser confirmado através dos resultados apresentados no $V C 1$, onde o $R^{2}$ do modelo foi pequeno.

\section{Conclusões}

O Programa Bolsa Família foi criado em 2003 no Brasil disponibilizando várias ações de transferência de renda para as pessoas de baixa renda e mais carentes. No início, o Programa beneficiava cerca de 327 mil famílias (2004), alcançando, em 2016, cerca de 14 milhões de famílias brasileiras.

Uma das críticas encontradas na literatura é o possível efeito negativo deste programa ao estimular a permanência de parte dos trabalhadores no mercado informal brasileiro, onde não há visibilidade das remunerações, o que torna possível a manutenção do acesso à bolsa família. Desta forma, ao trabalhar no mercado informal, os trabalhadores se qualificam para acessar ao Programa Bolsa Família, viabilizando receber ou continuar recebendo os benefícios.

Este estudo inova ao analisar a relação entre o benefício do Bolsa Família e o número de trabalhadores no mercado informal em séries de tempo (mensais) de janeiro de 2004 a fevereiro de 2016, diferentemente dos trabalhos anteriores, que comparam dados de dois períodos consecutivos (Painel). Além disso, a pesquisa utiliza-se do modelo de estimação, o VEC, que permite estudar as relações de curto e longo prazo entre o Programa Bolsa Família e o mercado laboral informal.

Os resultados do estudo mostram que existe uma relação causal e positiva entre Bolsa Família e Mercado Laboral Informal; entre Desemprego e Mercado Laboral Informal; e entre Desemprego e Bolsa Família. Desta forma, em períodos econômicos recessivos, o maior número de desempregados tem o efeito de aumentar o número de trabalhadores no mercado informal e o número de pessoas com Bolsa Família. Esta relação já era esperada para momentos de menor crescimento econômico e de maior taxa de desemprego na medida em que existe uma redução da renda e precarização dos postos de trabalho nestes períodos. Todavia, cabe aqui destacar a importância do Bolsa Família como uma política pública que permite uma compensação a população mais pobre e carente, principalmente nos momentos de maior fragilidade econômica e social.

Especificamente com relação à Bolsa Família e ao Mercado Laboral Informal, apesar de existir uma relação positiva e causal entre as variáveis, o efeito do Programa Bolsa Família sobre o Mercado Laboral Informal mostrou-se permanente, mas pequeno. Assim, ainda que a Bolsa Família possa estimular a migração ao mercado laboral informal, existem outras variáveis e aspectos estruturais na economia brasileira que explicam a dimensão expressiva do mercado informal brasileiro. Considerando aspectos mencionados anteriormente, o emprego informal representava cerca de $57 \%$ da força de trabalho no Brasil, segundo os dados da PNAD, 2008.

Portanto, este trabalho avança na análise de efeitos do Programa Bolsa Família e o mercado laboral informal, utilizando o VEC, que permite estudar a relação das variáveis. É importante enfatizar que trabalhos anteriores destacaram que os benefícios do PBF causam efeitos positivos no mercado laboral informal e este 
trabalho aponta que existe uma relação pequena entre PBF e o Mercado Laboral Informal, embora positiva e persistente no tempo.

\section{REFERÊNCIAS}

AHMAD, E. BEST, M. Financing Social Policy in the Presence of Informality. Working Paper. Londres: IGC - International Growth Centre - LSE e Univ. of Oxford, 2012.

BARBOSA, A. L. N. H. CORSEUIL, C. H. L. Bolsa Família, Escolha Ocupacional e Informalidade no Brasil. Boletim Mercado de Trabalho - Conjuntura e Análise. Nota Técnica/ed. IPEA, v. 47, p. 29-35, 2011.

BARBOSA, A. L. N. H. CORSEUIL, C. H. L. Bolsa Família, escolha ocupacional e informalidade no Brasil. Texto para Discussão 1948. Rio de Janeiro: IPEA, 2014.

BID (Banco Interamericano de Desenvolvimento). O fim da pobreza hereditária, 2009. Disponível em: http://www.iadb.org/pt/noticias/artigos/2009-09-02/o-fim-dapobreza-hereditaria,5557.html. Acesso: 24 de julho de 2017.

BRAUW, A. et al. The Impact of Bolsa Família on Child, Maternal, and Household Welfare. AIBF - International Food Policy Research Institute, United Nations Development Program, Ministério de Desenvolvimento Social e Combate à Fome, 2012.

CARDOSO, E. E SOUZA, A. The impact of cash transfers on child labor and school attendance in Brazil. Nashville Working Paper, n. 4-W07, 2004.

CHEIN, F. ANDRADE, M. V. RIBAS, R. P. Políticas de transferência de renda e condição nutricional de crianças: uma avaliação do Bolsa Família. Texto para Discussão, n. 312, Cedeplar, Universidade Federal de Minas Gerais. 2007.

CORREA JUNIOR, C. B. TREVISAN, L. N. MELLO, C.H.P. Impactos do Programa Bolsa Família no mercado de trabalho dos municípios brasileiros. Revista de Administração Pública. V.3, n. 5, p. 838-858, 2019. http://dx.doi.org/10.1590/0034761220180026.

CECCHINI, S. Transferências condicionadas na América latina e Caribe: da inovação à consolidação. In: CAMPELLO, Tereza; NERI, Marcelo C. (Org.). Programa Bolsa Família: uma década de inclusão e cidadania. Brasília: IPEA, 2013. p. 369-396. Disponível em:

https://www.researchgate.net/publication/258129635_Transferencias_Condicionada s_na_America_Latina_e_Caribe_Da_Inovacao_a_Consolidacao. Acesso: 8 de dezembro de 2020.

DIMITRIOS, A. HALL, S. G. Cointegration and Error-Correction Models. Applied Econometrics, a modern approach. Nova lorque: Palgrave McMillan, 2006.

FERRO, A. R. KASSOUF, A. L. LEVISON, D. The impact of conditional cash transfer programs on household work decisions in Brazil. In: Akee, R.K.Q., Edmonds, E.V. and Tatsiramos, K. (Ed.) Child Labor and the Transition between School and Work. 
Research in Labor Economics, v. 31. Emerald Group Publishing Limited, Bingley, pp. 193-218, 2010. https://doi.org/10.1108/S0147-9121(2010)0000031010.

FISZBEIN, A. SCHADY, N. Conditional Cash Transfeers: reducing present and future poverty. Washington, DC: The World Bank, 2009.

FOGUEL, M. N. F. BARROS, R. P. The effects of conditional cash transfer programmes on adult labour supply: an empirical analysis using a time-series-crosssection sample of Brazilian municipalities. Estudos Econômicos, v. 40, n. 2, p. 259293, 2010.

IBGE (Instituto Brasileiro de Geografia e Estatística). IBGE divulga as estimativas populacionais dos municípios em 2016. Diretoria de Pesquisas - DPE, Coordenação de População e Indicadores Sociais - COPIS, 2016. Disponível em:

https://agenciadenoticias.ibge.gov.br/. Acesso: 8 de dezembro de 2020.

JOHANNSEN, J. TEJERINA, L. GLASSMAN, A. Conditional Cash Transfer Programs in Latin America: Problems and Opportunities. BID - Banco Interamericano de Desenvolvimento. Disponível em: https://publications.iadb.org/handle/11319/2530. Acesso: 17 de outubro de 2017.

LEVY, S. Buenas intenciones, malos resultados: política social, informalidad y crecimiento económico en México. México: Oceano, 2010.

MDS (Ministérios do Desenvolvimento Social). Programa Bolsa Família - Benefícios. 2015. Disponível em: http://mds.gov.br/assuntos/bolsa-familia/o-que-e/beneficios. Acesso: 20 de março de 2017.

OLIVEIRA, L. F. B. SOARES, S. D. O que se sabe sobre os efeitos das transferências de renda sobre a oferta de trabalho. Rio de Janeiro: IPEA - Texto para discussão $1738,2012$.

OLIVEIRA, B. R. DAROIT, D. Public Policy Networks and the Implementation of the Bolsa-Família Program: An Analysis Based on the Monitoring of School Attendance. Education Policy Analysis Archives, v. 28, n. 120, p. 1-27, 2020. https://doi.org/10.14507/epaa.28.4499.

PEDROZO E. Uma avaliação ex-ante dos impactos do Bolsa Família na redução do trabalho infantil. Encontro Nacional de Economia, 35. ANPEC. 2007.

RIBAS, R. P. E SOARES, F.V. Is the effect of conditional transfers on labor supply negligible everywhere? 6th IZA/World Bank Conference: Employment and Development. Mexico: [s.n.], 2011.

SAAVEDRA, J.E. The Effects of Conditional Cash Transfer Programs on Poverty Reduction, Human Capital Accumulation and Wellbeing. Disponível em: http://www.un.org/esa/socdev/egms/docs/2016/Poverty-SDGs/JuanSaavedrapaper.pdf. Acesso: 8 de dezembro de 2020.

SANTOS, D. B. et al. Os efeitos do Programa Bolsa Família sobre a duração do emprego formal dos indivíduos de baixa renda. Revista de Administração Pública, v.51, n.5, 708-733, 2017. https://doi.org/10.1590/0034-7612171851. 
SEGURA-PÉREZ, S. GRASEDA, R. PÉREZ-ESCAMILLA, R. Conditional cash transfer programs and the health and nutrition of Latin American children. Revista

Panamericana de Salud Publica, v. 40, n.2, p. 124-137, 2016.

https://www.scielosp.org/pdf/rpsp/2016.v40n2/124-137/en.

SILVA, G. M. Mercado de Trabalho e Bolsa Família: políticas de emprego e inserção laboral dos beneficiários do Distrito Federal (2015-2018). Dissertação de Mestrado em Governança e Desenvolvimento da Escola Nacional de Administração Pública. Brasília, 2019. Disponível em:

https://repositorio.enap.gov.br/bitstream/1/4340/1/Disserta\%C3\%A7\%C3\%A30\%20Gleici ane\%20Marcelino\%20da\%20Silva.pdf. Acesso: 8 de dezembro de 2020 .

SKOUFIAS, E. DI MARO, V. Conditional Cash Transfers, Adult Work Incentives, and Poverty. Impact Evaluation Series n. 5. Washington: Bank's Policy Research Working Paper Series, 2006.

SOARES, F. V. RIBAS, R. P. OSÓRIO, R. G. Evaluating the Impact of Brazil's Bolsa Família: Cash Transfer Programmes in Comparative Perspective. Latin American Research Review, v. 45, n. 2, p. 173-190, 2010.

SOARES, S. et al. Os impactos do benefício do programa Bolsa Família sobre a desigualdade e a pobreza. Bolsa Família 2003-2010: avanços e desafios. Brasilia: IPEA, v.2, 2011.

STAMPINI, M. TORNAROLLI L. The growth of conditional cash transfers in Latin America and the Caribbean: did they go too far? Inter-American Development Bank, Policy brief No. IDB-PB-185. 2012.

SUGIYAMA, N. B. The diffusion of Conditional Cash Transfer programs in the Americas. Global Social Policy, v.11, p. 250-278, 2011. https://doi.org/10.1177/1468018111421295.

TAVARES, P. A. Efeito do Programa Bolsa Família sobre a oferta de trabalho. XIII Seminário sobre Economia Mineira. In: Economia, História, Demografia e Políticas Públicas. Diamantina, MG: [s.n.], 2008.

TEIXEIRA, C. G. Efeitos da transferência de renda na oferta de trabalho. Boletim Mercado de Trabalho. Conjuntura e Análise, n. 46, Rio de Janeiro: IPEA, p. 37-43, 2011.

Augusto Mussi Alvim. Doutor em Economia. PUCRS. Professor Titular. Av. Ipiranga, 6681. Bairro Partenon, Porto Alegre, RS.

E-mail augusto.mussi.alvim@pucrs.br.

Carlos Godward. Mestre em Economia do Desenvolvimento pela PUCRS. Dermalaser Dermatologia Soc. Com. Ltda. Gerente Financeiro. Rua Prof. Ulisses Cabral, 566 - 91330-520, Porto Alegre, RS. E-mail cdgodward@gmail.com. 
Como citar: ALVIM, Augusto Mussi; GODWARD, Carlos. O Programa Bolsa Família Estimula a Permanência dos Trabalhadores Brasileiros de Baixa Renda no Mercado Informal?. Redes (St. Cruz Sul, Online), Santa Cruz do Sul, v. 25, p. 2227-2250, 2020. ISSN 1982-6745. doi:https://doi.org/10.17058/redes.v25i0.14011.

\section{CONTRIBUIÇÃO DE CADA AUTOR}

a. Fundamentação teórico-conceitual e problematização: Carlos Godward e Augusto M. Alvim

b. Pesquisa de dados e análise estatística: Carlos Godward e Augusto M. Alvim

c. Elaboração de figuras e tabelas: Carlos Godward

d. Elaboração e redação do texto: Carlos Godward e Augusto M. Alvim

e. Seleção das referências bibliográficas: Carlos Godward e Augusto M. Alvim

Fontes de financiamento: CAPES/PROSUP. 